\title{
Psychological Interventions for Women with Persistent Pelvic Pain: A Survey of Mental Health Clinicians
}

\author{
Tiffany Brooks (D) \\ Rebecca Sharp' \\ Susan Evans (iD ${ }^{2}$ \\ John Baranoff ${ }^{3}$ \\ Adrian Esterman (iD) \\ 'The University of South Australia, \\ Clinical and Health Sciences, Adelaide, \\ South Australia, Australia; ${ }^{2}$ Welland \\ Health, North Adelaide, South Australia, \\ Australia; ${ }^{3}$ Centre for Treatment of \\ Anxiety and Depression, Adelaide, South \\ Australia, Australia
}

\begin{abstract}
Objective: To establish which psychological therapies mental health professionals use with reference to the treatment of women with persistent pelvic pain conditions. This research investigates overall therapies and specific techniques that clinicians believe are the most effective with this patient group, and the challenges mental health clinicians face in administering interventions. The study aims to suggest improvements to clinical practice and establish directions for targeted future research.
\end{abstract}

Design: Cross-sectional survey design.

Methods: An online survey was developed to ask mental health clinicians questions regarding the therapies and techniques they use with women experiencing persistent pelvic pain, their perspective on their practice in this area. The survey was advertised on relevant social media and professional websites. Survey results were tabled, and chi-square statistical analyses were undertaken to examine differences in therapy use according to country and profession.

Results: Mental health clinicians predominantly utilized cognitive behavioral therapy, acceptance and commitment therapy and associated techniques for women with persistent pelvic pain conditions. The results of the chi-square analyses showed that psychologists were more likely to use cognitive behavioral therapy or acceptance and commitment therapy, than counsellors who preferred counselling interventions. Chi-square analyses showed that Australian clinicians used acceptance and commitment therapy with a higher frequency than mental health clinicians in other countries. Clinicians provided multiple insights into their experiences working with women affected by persistent pelvic pain and their opinions as to valuable future research directions.

Conclusion: Cognitive behavioral therapy, acceptance and commitment therapy, and mindfulness therapies were most commonly used by mental health clinicians working with women with persistent pelvic pain conditions, despite severely limited evidence for the use of these psychological interventions in this client group.

Keywords: persistent, pelvic, pain, psychology, therapy, clinicians

\section{Plain Language Summary}

1. Mental health therapy is often recommended to women with persistent pelvic pain. This is to assist with the impact of the condition and to help with other mental health conditions that may be present, such as anxiety or depression.

2. Documenting what mental health therapies and techniques work for women who have persistent pelvic pain is challenging, due to overlapping health conditions and limited research. 
3. Our research determined that psychologists were more likely to use cognitive behavioral therapy and acceptance and commitment therapy, whereas counsellors preferred to use counselling therapy.

4. Our study found that Australian psychologists were more likely to use acceptance and commitment therapy than clinicians in other countries.

5. Clinicians gave insights into their experience working with women who have persistent pelvic pain.

6. The results of this study will help inform useful directions for the future research needed to improve clinical practice and therapy outcomes for women with persistent pelvic pain.

\section{Introduction}

Up to $26 \%$ of women worldwide experience Persistent Pelvic Pain (PPP), which is not solely related to their menstrual cycle. ${ }^{1}$ PPP, also known as chronic pelvic pain, is defined as pain in the pelvic region that has persisted for over three months. ${ }^{2}$ It is regarded as different from pain related to intercourse or period pain and can be due to biological conditions or no biological cause., ${ }^{2,3}$

PPP conditions cost the Australian economy approximately $\$ 6.5$ billion dollars in economic losses due to aspects such as health care utilization and reduced productivity. ${ }^{4}$ Further, the estimated economic cost to an individual of having a PPP condition may be up to $\$ 20$, 898 per annum. ${ }^{4}$ Women with these conditions experience mental health concerns, restricted activities, reduced quality of life and wellbeing, when compared to the general population. $^{5-7}$

Organizations that are responsible for providing guidelines for the appropriate treatment of people with persistent pain, such as the International Association for the Study of Pain and the Australian Pain Society, recommend that mental health be considered as part of multidisciplinary pain management according to the personcentered biopsychosocial model. ${ }^{8,9}$ This recommendation is due to both the psychological impact of experiencing persistent pain conditions, as well as the existence of comorbid mental health conditions, which can impact on pain management outcomes. ${ }^{9}$ The person-centered biopsychosocial model specifies that biological, psychological, social and contextual factors and the overlap between them must be considered in order to provide the best treatment for musculoskeletal conditions, such as PPP. ${ }^{10}$

Despite the prevalence and psychological impact of PPP, an optimal mental health treatment strategy for this condition is yet to be defined. There are three key challenges with reference to arriving at a consensus on effective mental health intervention for women with PPP. These include the absence of quality evidence as to the effectiveness of mental health interventions for women with PPP, overlap between PPP and co-morbid mental and physical health conditions, and limited information as to what mental health providers view as barriers to adequate care.

Recent reviews have concluded that there is a lack of high-quality evidence regarding predictors of mental health outcomes and efficacy of mental health interventions for women with PPP conditions. ${ }^{11-13}$ Currently there is a severe lack of quality studies with reference to what assessment measures, education concepts and mental health interventions are effective for use with women who have PPP. ${ }^{14}$

Although there are many recent studies that have investigated components of pain education that are valuable for use with other chronic pain conditions, such as fibromyalgia and spinal pain, ${ }^{14-16}$ there is limited information as to what is specifically useful for women with PPP. For example, Cognitive Behavioral Therapy (CBT) in patients with persistent low back pain is evidence based. ${ }^{17,18}$ A review by Richmond et $\mathrm{al}^{18}$ found that across 23 studies with 3359 participants, who had persistent lower back pain, CBT improved pain and disability ratings. A study by Alpattu and Bishop ${ }^{19}$ found that the fear-avoidance model of therapy used in CBT appeared to have relevance to treating psychological factors for women with PPP conditions though they did not test it with women with these conditions. Hypnosis, particularly with the inclusion of analgesic suggestions, has also been shown to lead to reductions in pain severity and improved function, for groups of clients with conditions such as persistent neck and lower back pain. ${ }^{20,21}$ Acceptance and commitment therapy (ACT) is building an evidence-base for improving outcomes in chronic pain conditions. Improvements in psychological flexibility following ACT have been confirmed in groups of patients with chronic neck pain and fibromyalgia. Proper validation studies are required before ACT can emerge as the gold-standard therapy for the management of persistent pain conditions. ${ }^{22,23}$ The lack of evidence on mental health assessment and intervention for women with PPP means that mental health professionals working in this area must either draw from the limited evidence available within the literature or rely on techniques used for co-morbid physical or mental health conditions, that are not proven for PPP. 
This may not seem problematic, due to the recent discussions surrounding Chronic Overlapping Pain Conditions (COPCs). The National Institutes of Health has recognized a series of pain conditions and disorders that commonly occur together called COPCs. ${ }^{24}$ COPCs include conditions and symptoms commonly reported by women who have PPP conditions, including endometriosis, chronic headaches, Irritable Bowel Syndrome (IBS), chronic fatigue, chronic lower back pain and interstitial cystitis. ${ }^{25}$ Persons with COPCs have been shown to have a higher risk of developing co-morbid mental health conditions, including depression and anxiety. ${ }^{25}$ These aspects of mental health also have strong potential to influence coping skills and the management of pain associated with COPCs. The overlap of physical and mental health conditions for COPCs means that there are most likely some shared as well as some unique psychological symptoms and risk factors. ${ }^{25}$ Therefore, it makes sense that mental health interventions that work for other conditions associated with COPCs, such as lower back pain and IBS, may also be effective for women with PPP.

However, this does not take into account aspects of mental health or the pain experience that might be unique to women with PPP. For instance, each chronic pain condition has some specific physical features such as locus of pain, where pain sensitization occurs, and other conditionspecific symptoms, which have the potential to differentially impact on disability and mental health. A recent paper has noticed differences in pain sensitization mechanisms in the body and brain across different persistent pain conditions such as IBS and musculoskeletal conditions such as persistent lower back pain. ${ }^{26}$ Although the overlap of muscle and nerve activity in this area can mean women with PPP experience difficulties in these areas, ${ }^{27}$ this does not mean that the experience or cause of this and therefore the appropriate therapy to address the condition would necessarily be the same.

The presence of both shared and unique physical and psychological factors across COPCs make decisionmaking difficult for mental health clinicians working with women who have PPP. It is unclear what factors are shared or unique to women with PPP, and therefore difficult to decide what mental health interventions might prove effective to address mental health for these conditions. If clinicians are drawing multiple approaches and combinations of assessment, educational concepts and therapies, then it is also difficult to arrive at a consensus of what consistently works for women with PPP. Therefore, understanding the central mechanisms of mental health for PPP in relation to other COPCs, and as a chronic pain condition with unique features, is essential to the development of mental health interventions that work for this group of clients. ${ }^{28}$

One way to identify what shared or unique aspects of mental health interventions work for women with PPP is to survey mental health clinicians working in the area. This has the potential to identify what clinicians view as effective, but also establish barriers to appropriate care. A previous survey of medical providers who worked with PPP by Megowan et al. ${ }^{29}$ found that limited evidence base and consequently vague assessment and treatment directives made it more challenging for providers to work with PPP clients in comparison to clients with other COPCs such as IBS. Medical providers have also reported that the absence of evidence based guidelines, limited support for clinicians and the emotional toll of working with women who have PPP are key barriers to providing appropriate care. ${ }^{30}$ There is also limited information as to what challenges mental health care workers experience when working with clients who have PPP and what future research might address these barriers.

This study addresses this knowledge gap within the current literature using a series of questions to focus on three key aims: (1) to identify the psychological therapies used in clinical practice by mental health clinicians. This includes clinician views as to the efficacy of specific treatments; (2) to establish clinician attitude and experience using these therapies in clinical practice and record their opinions on the benefits and difficulties encountered when working with this patient population; (3) to establish clinical opinions as to where future research priorities should be directed. The aims were developed from the research literature and formulated with assistance from specialists working with women who have PPP.

\section{Methods}

\section{Survey Development}

A draft bank of survey questions was sent to three experts with specific knowledge across the areas of chronic pain conditions, pelvic pain, psychology and gynecology for validation. This included a senior clinical psychologist working with chronic pain groups, a prominent professor of physiotherapy specializing in chronic pain research, and a specialist gynecologist and pain medicine physician who 
has been working with women who have PPP for over 20 years.

Each expert reviewed questions individually for face validity and marked them with one of the following three responses: "not valid", "unsure" or "valid". If all three experts marked a question as valid it was then included in the final survey draft. Questions with disagreement across experts were discussed by the research team to address whether they should be included. The panel was also asked to assess the content validity of the questions as a whole and all agreed that the questions had good content validity. Of the 56 questions in the survey draft presented to the experts, they disagreed as to the inclusion of 5 proposed questions once edits to question wording had been made. The questions related to asking participants about the following: whether family is included in therapy sessions; whether family was included if a client was under 18: whether neuroscience pain education should be included in therapy; whether consent was sought formally for communication with other professionals; and the distinction between psychological processes and concerns. These questions were then discussed by the research team. Family inclusion and under 18 questions were excluded as they were deemed outside of the study scope. The neuroscience pain education question was merged with a different question on psycho-education provided in therapy. A separate question surrounding consent was created, which divided into signed, verbal, and no consent as separate options. The question on processes and concerns was kept, with more options for clinicians to select in both processes and concerns aspects.

The survey draft, including information sheet, consent form and questions, were then developed in an online format using SurveyMonkey ${ }^{31}$ software. The draft online survey was then sent to eight professionals working in the areas of mental health and chronic pain, in order to establish test-retest reliability. The research team identified thirty registered psychologists who publicized that they worked with clients who clinically had persistent pain and mental health-related concerns in Australia. All thirty were sent an email asking them to take part in the testretest component of this study, with eight agreeing to take part.

The clinicians surveyed for test-retest reliability were sent the survey in SurveyMonkey ${ }^{31}$ online software format for completion on two occasions that were seven to ten days apart. Results from the test-retest were then loaded into MedCalc Statistical Software ${ }^{32}$ and inter-rater agreement weighted Kappa conducted for each question. All items had a Kappa of greater than 0.6 (range 0.6-1). The survey was then given to two clinicians, a clinical psychologist and a pain physician, for pre-testing. Both clinicians went through the survey step by step with the researchers and provided in-depth feedback as to the format and questions. Changes to the font size, response box formats, colours used and errors made to skip question logic were all corrected from their feedback.

The final version of the survey containing 51 questions was then edited in SurveyMonkey ${ }^{31}$ and took approximately 30 minutes for participants to complete. The first section of the survey collected the demographic information from participants and screened for our inclusion criteria, with yes or no format questions such as "I am a mental health clinician" and "I work with women who have persistent pelvic pain" Then questions were asked using either 10-point Likert-scale responses or multiple choice tick box formats. An example of a Likert-scale response was "How confident are you using mental health interventions and techniques with women? (please rate on the slider below with " 0 " being not confident at all and "10" being very confident)". An example of the multiple choice question format was "Do you use any of the following therapies when working with women who have persistent pelvic pain? (please tick all that apply)". This question was followed by a list of common therapies, of which clinicians could select multiple options, and space for clinicians to provide additional responses in an "other" box.

\section{Procedure}

Once the final version was completed, the survey was promoted using social media pages and professional websites. This included the Australian Psychological Society and Pelvic Pain Foundation of Australia. Clinicians who listed themselves as working with chronic pain, women's health, and pelvic pain client groups publically listed on the "find a psychologist" service on the Australian Psychological Society website or through google search were contacted via email and invited to participate. Potential participants clicked on the link to the online survey where they were directed through the information sheet and consent form. Participants were not permitted to continue through the survey or submit responses if they declined consent to participate. Consenting participants were then guided through questions across the following key areas: demographic information, psychological 
theories, interventions and techniques used with women with chronic pelvic pain, knowledge of the evidence base, experiences in practice, and views on future research directions. Question formats included Likert scales, multiple choice and free response formats. Participation was voluntary and respondents were not offered financial incentives to participate. Participants were offered an option to contact the researchers to obtain a copy of the final results for the study on the final page of the survey, in order to keep their contact details and results request separate from their anonymous responses.

\section{Inclusion Criteria}

The study population were mental health clinicians who worked with adult females who met criteria for PPP. The formal definition of PPP was clarified in the information sheet and consent form of the survey so that clinicians could ensure they worked with this population of interest in this study. Participants were included if they were 18 years or over, worked clinically providing mental health services to women with PPP, and gave formal consent to their participation.

\section{Exclusion Criteria}

There were no exclusion criteria.

\section{Ethics Approval}

This survey and all materials used were approved by the University of South Australia Human Research Ethics Committee on 01/09/2019, ethics application number 202576.

\section{Data Availability Statement}

The data set and materials for this study have been made available on Open Science Framework accessible at https://mfr.osf.io/render?url=https $\% 3 \mathrm{~A} \% 2 \mathrm{~F} \% 2 \mathrm{Fosf.io} \%$ 2Ft2f7m\%2Fdownload.

\section{Data Collection}

Data collection was conducted between 5th of April and 3rd of September 2020.

\section{Data Analysis}

With respect to sample size, our intention was to obtain 96 responses for the study as a whole. This provides a margin of error of at least $\pm 10 \%$ margin with $95 \%$ confidence. The completion rate for the online survey was $68 \%$. The final survey data were transferred from SurveyMonkey ${ }^{31}$ survey to $\mathrm{SPSS}^{33}$ software for analysis. Data analysis was primarily descriptive, with counts and percentages provided. Chisquare analyses were conducted to explore the results by some features of the respondents.

\section{Results}

\section{Demographic results}

Ninety-six completed and usable questionnaires were obtained. Demographic information for the participants is provided in Table 1.

The majority of clinicians that participated were female, aged between 35 and 49. A majority of therapists worked full-time (35 hours or more per week) in private clinic settings. Most clinicians resided in Australia,

Table I Age, Gender and Work Setting of Participants

\begin{tabular}{|c|c|c|c|}
\hline & Category & $\mathbf{N}$ & $\%$ \\
\hline \multirow[t]{10}{*}{ Age } & $25-29$ & 5 & 5.2 \\
\hline & $30-34$ & 9 & 9.4 \\
\hline & $35-39$ & 12 & 12.5 \\
\hline & $40-44$ & 20 & 20.9 \\
\hline & $45-49$ & 14 & 14.6 \\
\hline & $50-54$ & 8 & 8.3 \\
\hline & $55-59$ & 8 & 8.3 \\
\hline & $60-64$ & 8 & 8.3 \\
\hline & $65+$ & 12 & 12.5 \\
\hline & Total & 96 & 100 \\
\hline \multirow[t]{3}{*}{ Gender } & Female & 90 & 93.8 \\
\hline & Male & 6 & 6.2 \\
\hline & Total & 96 & 100 \\
\hline \multirow[t]{6}{*}{ Country } & Australia & 42 & 43.8 \\
\hline & United Kingdom & 20 & 20.8 \\
\hline & Canada & 13 & 13.5 \\
\hline & Other & 14 & 14.6 \\
\hline & New Zealand & 7 & 7.3 \\
\hline & Total & 96 & 100 \\
\hline \multirow[t]{7}{*}{ Work setting } & Solo private practice & 32 & 33.3 \\
\hline & Group private practice & 21 & 21.9 \\
\hline & Hospital & 6 & 6.3 \\
\hline & $\begin{array}{l}\text { Public state based and solo private } \\
\text { practice }\end{array}$ & 6 & 6.3 \\
\hline & Public health and state based hospital & 5 & 5.2 \\
\hline & Other settings & 26 & 27 \\
\hline & Total & 96 & 100 \\
\hline \multirow[t]{4}{*}{ Work Hours } & Full time (35+ hours pw) & 48 & 50.0 \\
\hline & Part time (10-35 hours pw) & 44 & 45.8 \\
\hline & Occasional (0-9 hours pw) & 4 & 4.2 \\
\hline & Total & 96 & 100 \\
\hline
\end{tabular}

Abbreviations: N, sample size; \%, percentage; pw, per week. 
although a large proportion resided in either the United Kingdom or Canada.

Most clinicians were self-described as Clinical Psychologists (28.1\%), Registered Psychologists (15.6\%) or Psychosexual Therapists that were also Counsellors (13.5\%). The most common qualifications that clinicians held were a Masters degree (17.7\%) or a doctorate degree $(16.7 \%)$. Half of the clinicians who participated had worked with clients who reported PPP for 9 years or more $(50.5 \%)$. The clinicians reported that on average one third of their case load of clients reported experiencing PPP ( $M=27.67, S D=23.59)$. Most clinicians rated their confidence in treating women with CPP as moderate to high on a Likert scale of 0 (not confident at all) to 10 (very confident) $(\mathrm{M}=68.54, \mathrm{SD}=21)$.

\section{Mental Health Assessment Tools}

The mental health clinicians surveyed reported the use of 51 different questionnaires with women who had PPP for assessment purposes. A list of common measures was provided to choose from in the online survey, as well as a free text box to add any they used that were not listed. The most commonly used questionnaires were the Depression Anxiety and Stress Scale -21 item (DASS$21),{ }^{34}$ the Pain Catastrophizing Scale (PCS) ${ }^{35}$ and the Pain Self-Efficacy Questionnaire ${ }^{36}$ (Table 2). Seventy two percent of clinicians conducted suicide risk assessment when working with women who had PPP. The mental health clinicians reported that Generalised Anxiety Disorder (GAD), Major Depressive Disorder, and PostTraumatic Stress Disorder (PTSD) were the most common co-morbid conditions (Table 2).

\section{Communication with Other Health Professionals}

Forty-six percent of clinicians reported that they regularly communicated with other health professionals with reference to their female clients with PPP. They were most commonly in contact with general practitioners (46.5\%), gynaecologists $(30.2 \%)$, pain specialists $(18.6 \%)$, pelvic (18.6\%) and regular physiotherapists $(39.5 \%)$. The clinicians that communicated with other professionals used telephone calls $(69.8 \%)$, emails $(62.8 \%)$ and letters (55.8\%). Most practitioners (97.8\%) sought consent from their clients to discuss their sessions with other clinicians, using signed (49.5\%) or verbal (54.7\%) consent processes, prior to communicating with other health professionals.
Table 2 Assessment Techniques Used During Mental Health Consultations

\begin{tabular}{|c|c|c|c|}
\hline & Category & $\mathbf{N}$ & $\%$ \\
\hline \multirow[t]{9}{*}{ Assessment measures used } & DASS-2I & 26 & 29.8 \\
\hline & PCS & 20 & 23.0 \\
\hline & PSQ & 12 & 13.8 \\
\hline & BPI & 5 & 5.8 \\
\hline & DASS-42 & 5 & 5.8 \\
\hline & PHQ-9 & 5 & 5.8 \\
\hline & GADS-7 & 3 & 3.4 \\
\hline & Other & II & 12.6 \\
\hline & Total & 87 & 100 \\
\hline \multirow[t]{3}{*}{ Risk Assessment Conducted } & Yes & 66 & 72.5 \\
\hline & No & 25 & 27.5 \\
\hline & Total & 91 & 100 \\
\hline \multirow{9}{*}{$\begin{array}{l}\text { Mental Health Conditions } \\
\text { seen co-morbidly with PPP } \\
\text { (multiple response, N=88). } \\
\text { Percentages are percentages } \\
\text { of respondents, so add up to } \\
\text { more than } 100 \%\end{array}$} & $\begin{array}{l}\text { Generalised Anxiety } \\
\text { Disorder }\end{array}$ & 62 & 70.5 \\
\hline & $\begin{array}{l}\text { Major Depressive } \\
\text { Disorder }\end{array}$ & 48 & 54.5 \\
\hline & $\begin{array}{l}\text { Post-Traumatic Stress } \\
\text { Disorder }\end{array}$ & $4 I$ & 46.6 \\
\hline & $\begin{array}{l}\text { Somatic Symptom } \\
\text { Disorder (Other } \\
\text { chronic pain condition) }\end{array}$ & 28 & 31.8 \\
\hline & $\begin{array}{l}\text { Borderline Personality } \\
\text { Disorder }\end{array}$ & 23 & 26.1 \\
\hline & Anorexia Nervosa & 10 & 11.4 \\
\hline & $\begin{array}{l}\text { Somatic Symptom } \\
\text { Disorder (not chronic } \\
\text { pain) }\end{array}$ & 10 & II.4 \\
\hline & $\begin{array}{l}\text { Avoidant Personality } \\
\text { Disorder }\end{array}$ & 9 & 10.2 \\
\hline & Bulimia & 9 & 10.2 \\
\hline
\end{tabular}

Abbreviations: N, sample size; \%, percentage; PPP, Persistent Pelvic Pain; DASS21, Depression Anxiety and Stress Scale - 2l item; PCS, Pain Catastrophizing Scale, PSQ, Pain Self-Efficacy Questionnaire; BPI, Brief Pain Inventory; DASS-42, Depression, Anxiety and Stress Scale 42-item; PHQ-9; Patient Health Questionnaire 9-item; GADS-7, Generalised Anxiety Disorder Scale 7-item.

\section{Clinician Views on Effective Therapies and Techniques}

Clinicians rated what therapies that they most commonly used, found most effective and believed to be most evidence based in Table 3. Clinicians selected therapy options and techniques from a list provided in the online survey, and provided additional therapies used in an open response box 
Table 3 Therapies Used by Mental Health Clinicians for Women with PPP and Their View on the Strength of Evidence for These

\begin{tabular}{|c|c|c|c|}
\hline & Category & $\mathbf{N}$ & $\%$ \\
\hline \multirow{7}{*}{$\begin{array}{l}\text { Strength of Evidence on } \\
\text { psychology use for PPP }\end{array}$} & Very strong & 18 & 18.8 \\
\hline & Strong & 37 & 38.5 \\
\hline & Average & 31 & 32.3 \\
\hline & I am not sure & 6 & 6.3 \\
\hline & Weak & 3 & 3.1 \\
\hline & Very weak & I & I \\
\hline & Total & 96 & 100 \\
\hline \multirow{18}{*}{$\begin{array}{l}\text { Therapies used (Multiple } \\
\text { response, } \mathrm{N}=91 \text { ). } \\
\text { Percentages are percentages } \\
\text { of respondents, } \\
\text { so add up to more than } 100 \%\end{array}$} & $\begin{array}{l}\text { Cognitive Behavioural } \\
\text { Therapy }\end{array}$ & 69 & 75.8 \\
\hline & $\begin{array}{l}\text { Acceptance and } \\
\text { Commitment Therapy }\end{array}$ & 52 & 57.1 \\
\hline & Counselling & 38 & 41.8 \\
\hline & Behavioural Therapy & 23 & 25.3 \\
\hline & Cognitive Therapy & 23 & 25.3 \\
\hline & Emotional & 20 & 21.9 \\
\hline & Reassurance & & \\
\hline & Psychodynamic & 19 & 20.9 \\
\hline & $\begin{array}{l}\text { Dialectical Behaviour } \\
\text { Therapy }\end{array}$ & 17 & 18.7 \\
\hline & EMDR & 16 & 17.6 \\
\hline & Prolonged Exposure & 13 & 14.3 \\
\hline & Schema Therapy & 12 & 13.2 \\
\hline & Narrative Therapy & 11 & 12 \\
\hline & $\begin{array}{l}\text { Cognitive Processing } \\
\text { Therapy }\end{array}$ & 10 & 10.9 \\
\hline & Cognitive Reassurance & 10 & 10.9 \\
\hline & Gestalt Therapy & 8 & 8.8 \\
\hline & Family Therapy & 8 & 8.8 \\
\hline & Psychosexual therapy & 5 & 5.5 \\
\hline \multirow{9}{*}{$\begin{array}{l}\text { Most effective therapy } \\
\text { (Multiple response, } \mathrm{N}=84 \text { ) }\end{array}$} & I do not know & 29 & 34.5 \\
\hline & $\begin{array}{l}\text { Cognitive Behavioural } \\
\text { Therapy }\end{array}$ & II & 13.0 \\
\hline & $\begin{array}{l}\text { Acceptance and } \\
\text { Commitment Therapy }\end{array}$ & 10 & 11.9 \\
\hline & $\begin{array}{l}\text { Combined therapeutic } \\
\text { approaches }\end{array}$ & 9 & 10.7 \\
\hline & Counselling & 4 & 4.7 \\
\hline & EMDR & 4 & 4.7 \\
\hline & $\begin{array}{l}\text { Psychosexual } \\
\text { approach }\end{array}$ & 4 & 4.7 \\
\hline & Other & 13 & 15.8 \\
\hline & Total & 84 & 100 \\
\hline
\end{tabular}

(Continued)
Table 3 (Continued).

\begin{tabular}{|l|l|r|r|}
\hline & Category & $\mathbf{N}$ & $\%$ \\
\hline Most evidence-based & Cognitive Behavioural & 57 & 62.6 \\
therapies & Therapy & & \\
\cline { 2 - 4 } $\begin{array}{l}\text { Multiple response, N=91) } \\
\text { Percentages are percentages } \\
\text { of respondents, so add up to } \\
\text { more than 100\% }\end{array}$ & I do not know & 29 & 31.9 \\
\cline { 2 - 4 } & Acceptance and & 27 & 29.7 \\
& Commitment Therapy & & \\
\cline { 2 - 4 } & Counselling & 9 & 9.9 \\
& Behavioural Therapy & 7 & 7.7 \\
& EMDR & 6 & 6.6 \\
& Emotional reassurance & 6 & 6.6 \\
\hline
\end{tabular}

Abbreviations: N, sample size; \%, percentage; EMDR, Eye Movement Desensitization and Reprocessing Therapy; PPP, Persistent Pelvic Pain.

provided. All therapists that responded used multiple therapeutic modalities. The most common therapeutic approaches used were mindfulness, Cognitive Behavioral Therapy (CBT), and Acceptance and Commitment Therapy (ACT). $\mathrm{CBT}$ and $\mathrm{ACT}$ were also rated as the most effective and evidence-based therapies used by clinicians. Despite these opinions, almost a third of clinicians reported that that they were unsure which therapies were the most evidence-based for use with women with PPP conditions.

The most popular techniques used in therapy with women with PPP were mindfulness, challenging anxiety and pain-related beliefs, thought challenging and the explain pain book (Table 4). Only $10.9 \%$ said that they did not know what techniques were evidence-based for use with women who had PPP.

Most clinicians used relaxation techniques (Table 4), with progressive muscle relaxation, diaphragm breathing and relaxation breathing reported as most utilized and evidence based. However, fifty-six percent of clinicians were unsure which relaxation skills were evidence based. Most clinicians reported that they did not use hypnosis as a therapy for women with PPP. Those that did reported techniques used for induction, deepening and suggestions in a free text response that was included in our clinician survey. The inductions that they reported using included 
Table 4 Therapeutic Techniques Used with Women Who Have PPP and Clinical Opinion on Their Evidence Base and Effectiveness

\begin{tabular}{|c|c|c|c|}
\hline & Category & $\mathbf{N}$ & $\%$ \\
\hline \multirow{24}{*}{$\begin{array}{l}\text { Techniques used (Multiple response, } \\
\mathrm{N}=92 \text { ). Percentages are percentages } \\
\text { of respondents, so add up to more } \\
\text { than } 100 \%\end{array}$} & Mindfulness & 70 & 76 \\
\hline & $\begin{array}{l}\text { Challenging } \\
\text { anxiety beliefs }\end{array}$ & 63 & 68.5 \\
\hline & $\begin{array}{l}\text { Challenging pain } \\
\text { related beliefs }\end{array}$ & 62 & 67.4 \\
\hline & $\begin{array}{l}\text { Though } \\
\text { challenging }\end{array}$ & 57 & 61.9 \\
\hline & $\begin{array}{l}\text { Explain pain } \\
\text { resource }\end{array}$ & 57 & 61.9 \\
\hline & $\begin{array}{l}\text { Emotional } \\
\text { regulation skills }\end{array}$ & 53 & 57.6 \\
\hline & $\begin{array}{l}\text { Self-soothe } \\
\text { activities }\end{array}$ & 49 & 53.3 \\
\hline & $\begin{array}{l}\text { Emotional } \\
\text { coping skills }\end{array}$ & 46 & 50 \\
\hline & Increasing & 43 & 46.7 \\
\hline & Valued & & \\
\hline & Activities & & \\
\hline & Pacing activities & 43 & 46.7 \\
\hline & $\begin{array}{l}\text { Activity } \\
\text { scheduling }\end{array}$ & 39 & 42.4 \\
\hline & $\begin{array}{l}\text { Behavioural } \\
\text { experiment }\end{array}$ & 34 & 36.9 \\
\hline & $\begin{array}{l}\text { Behavioural } \\
\text { activation }\end{array}$ & 33 & 35.9 \\
\hline & $\begin{array}{l}\text { Cognitive } \\
\text { defusion }\end{array}$ & 32 & 34.8 \\
\hline & Problem solving & 31 & 33.7 \\
\hline & $\begin{array}{l}\text { Prioritizing } \\
\text { activities }\end{array}$ & 28 & 30.4 \\
\hline & $\begin{array}{l}\text { Graded task } \\
\text { assignment }\end{array}$ & 28 & 30.4 \\
\hline & $\begin{array}{l}\text { Values } \\
\text { clarification }\end{array}$ & 23 & 25 \\
\hline & Exposure & 20 & 21.7 \\
\hline & $\begin{array}{l}\text { Imagery } \\
\text { rescripting }\end{array}$ & 14 & 15.2 \\
\hline & $\begin{array}{l}\text { Metacognitive } \\
\text { work }\end{array}$ & 13 & 14.1 \\
\hline & Chair work & 13 & I4.1 \\
\hline \multirow{3}{*}{$\begin{array}{l}\text { Relaxation techniques used (Multiple } \\
\text { response, } \mathrm{N}=92 \text { ). Percentages are } \\
\text { percentages of respondents, so add } \\
\text { up to more than } 100 \%\end{array}$} & Progressive & 65 & 70.7 \\
\hline & $\begin{array}{l}\text { muscle } \\
\text { relaxation }\end{array}$ & & \\
\hline & $\begin{array}{l}\text { Diaphragm } \\
\text { breathing }\end{array}$ & 62 & 67.4 \\
\hline
\end{tabular}

(Continued)
Table 4 (Continued).

\begin{tabular}{|c|c|c|c|}
\hline & Category & $\mathbf{N}$ & $\%$ \\
\hline & $\begin{array}{l}\text { Relaxation } \\
\text { breathing }\end{array}$ & 61 & 66.3 \\
\hline & $\begin{array}{l}\text { Five senses } \\
\text { mindfulness } \\
\text { exercise }\end{array}$ & 55 & 59.8 \\
\hline & Yoga & 4 & 4.3 \\
\hline & $\begin{array}{l}\text { Pelvic floor } \\
\text { relaxation } \\
\text { exercise }\end{array}$ & 4 & 4.3 \\
\hline & Guided imagery & 4 & 4.3 \\
\hline & $\begin{array}{l}\text { Autogenic } \\
\text { relaxation }\end{array}$ & 3 & 3.3 \\
\hline \multirow{5}{*}{$\begin{array}{l}\text { Evidence based relaxation } \\
\text { techniques (Multiple response, } \\
\mathrm{N}=92 \text { ). Percentages are percentages } \\
\text { of respondents, so add up to more } \\
\text { than } 100 \%\end{array}$} & I do not know & 52 & 56.5 \\
\hline & $\begin{array}{l}\text { Progressive } \\
\text { muscle } \\
\text { relaxation }\end{array}$ & 20 & 21.7 \\
\hline & $\begin{array}{l}\text { Relaxation } \\
\text { breathing }\end{array}$ & 13 & 14.2 \\
\hline & $\begin{array}{l}\text { Diaphragm } \\
\text { breathing }\end{array}$ & 12 & 13 \\
\hline & $\begin{array}{l}\text { Five senses } \\
\text { mindfulness } \\
\text { exercise }\end{array}$ & 10 & 10.9 \\
\hline \multirow[t]{3}{*}{ Hypnosis used } & Yes & II & 12.5 \\
\hline & No & 77 & 87.5 \\
\hline & Total & 88 & 100 \\
\hline
\end{tabular}

Abbreviations: $\mathrm{N}$, sample size, \%, percentage.

counting, body scanning, attention focusing, staircase method, candle visualization, eye fixation, and breathingbased. The deepening exercises that they utilized included five senses mindfulness exercise, progressive body relaxation, focusing attention and visualization. The suggestions utilized included warm sensations, using a pain dial to dial down pain, glove anesthesia, regression, anesthesia, relaxation washing over the body, and visualization.

The key problems that mental health clinicians attempted targeted with their clients using these therapeutic interventions 
Table 5 Education Provided in Sessions

\begin{tabular}{|c|c|c|c|}
\hline & Category & $\mathbf{N}$ & $\%$ \\
\hline Pain education provided & $\begin{array}{l}\text { Yes } \\
\text { No } \\
\text { Total }\end{array}$ & $\begin{array}{r}80 \\
8 \\
88\end{array}$ & $\begin{array}{r}90.9 \\
9.1 \\
100\end{array}$ \\
\hline $\begin{array}{l}\text { Type of education provided } \\
\text { (Multiple response, } \mathrm{N}=88 \text { ). } \\
\text { Percentages are percentages of } \\
\text { respondents, so add up to more } \\
\text { than } 100 \%\end{array}$ & $\begin{array}{l}\text { Pain and the brain } \\
\text { Fear-avoidance } \\
\text { model } \\
\text { Bio-psycho-social } \\
\text { model } \\
\text { Central } \\
\text { sensitization } \\
\text { Neuroplasticity } \\
\text { Biology of } \\
\text { vulvodynia } \\
\text { Pacing activity } \\
\text { Referred to } \\
\text { others for pain } \\
\text { education } \\
\text { Biology of } \\
\text { Endometriosis } \\
\text { Directed to } \\
\text { resources } \\
\text { How a nerve } \\
\text { works } \\
\text { Peripheral } \\
\text { sensitization } \\
\text { Pacing energy } \\
\text { Inflammation } \\
\text { Nociception } \\
\text { Medicine cabinet } \\
\text { in the brain } \\
\text { Neuro-immune } \\
\text { interaction } \\
\text { Immune priming }\end{array}$ & $\begin{array}{l}25 \\
22 \\
21 \\
16\end{array}$ & $\begin{array}{r}32.9 \\
29.5 \\
28.4 \\
25.0 \\
23.9 \\
18.2 \\
11.4 \\
5.7\end{array}$ \\
\hline \multirow[t]{2}{*}{$\begin{array}{l}\text { Prevented from using pain } \\
\text { education (Multiple response, } \\
\mathrm{N}=8 \text { ) }\end{array}$} & $\begin{array}{l}\text { No training in the } \\
\text { area }\end{array}$ & 4 & 50 \\
\hline & $\begin{array}{l}\text { Not familiar with } \\
\text { the literature } \\
\text { No familiar with } \\
\text { the concepts } \\
\text { Total }\end{array}$ & 2 & $\begin{array}{r}25 \\
25 \\
100\end{array}$ \\
\hline $\begin{array}{l}\text { Would learn pain education if it } \\
\text { was easily accessible }\end{array}$ & $\begin{array}{l}\text { Yes } \\
\text { No } \\
\text { Total }\end{array}$ & $\begin{array}{l}8 \\
0 \\
8\end{array}$ & $\begin{array}{r}100 \\
0 \\
100\end{array}$ \\
\hline
\end{tabular}

(Continued)
Table 5 (Continued).

\begin{tabular}{|l|l|l|r|}
\hline & Category & $\mathbf{N}$ & $\%$ \\
\hline $\begin{array}{l}\text { Other types of education provided } \\
\text { (Multiple response, N=92). }\end{array}$ & Psycho-education & 77 & 83.7 \\
$\begin{array}{l}\text { Percentages are percentages of } \\
\text { respondents, so add up to more } \\
\text { than 100\% }\end{array}$ & $\begin{array}{l}\text { Education about } \\
\text { menstrual } \\
\text { difficulties } \\
\text { Education about } \\
\text { hormones }\end{array}$ & 37 & 40.2 \\
\hline
\end{tabular}

Abbreviations: $\mathrm{N}$, sample size; \%, percentage.

and techniques were inaccurate pain beliefs (89.1\%), anxious thinking $(86.9 \%)$, pain avoidance $(76 \%)$, difficulties with emotional regulation (70.7), and unprocessed trauma (65.2\%).

\section{The Inclusion of Pain Education in Consultations}

Eighty percent of clinicians included a form of pain education in their sessions for women with PPP (Table 5). Pain education topics covered included pain and the brain, the fear-avoidance model, the bio-psycho-social model, and central sensitization.

Where pain education was not included, 50\% described a lack of pain training as the reason for this omission. All of the clinicians who did not use pain education in sessions expressed an interest in learning more within this area if this education was made easily accessible for them. Other forms of education included in sessions included general psychoeducation, education around menstrual and hormonal symptoms.

\section{Use of Additional Resources During Consultations}

Most clinicians (92.5\%) used resources, software applications and supports during their sessions with women with PPP. These included online resources (67\%), books $(56.8 \%)$, and "explain pain" resources created by Moseley and Butler ${ }^{37}$ that explain the interaction between pain and the brain (39.8\%). The most commonly accessed 
supports for clinicians were professional development (59\%), peer supervision (59\%) and discussions with a team of clinicians $(40 \%)$. Forty-three percent of clinicians recommended electronic application software use for their clients for use with aspects of therapy including relaxation, mindfulness and symptom monitoring.

\section{Differences in Therapy Use Across Profession and Country}

The various professions were consistent with their use of behavioural therapy with women who had PPP. However, they differed significantly in their use of CBT $\left[X^{2}(d f=5\right.$, $\mathrm{N}=91)=42.75, p<0.001]$, ACT $\left[X^{2}(d f=5, \mathrm{~N}=91)=45.449\right.$, $p<0.001]$ and counselling therapies $\left[X^{2}(d f=5, \mathrm{~N}=91)\right.$ $=41.789, p<0.001]$. Psychologists and psychosexual therapists were more likely to used CBT than the counsellors and therapists from other clinicians. Psychologists tended to use ACT more than all other professions, including psychosexual therapists, counsellors and other professionals. Counselling and psychosexual therapists with counsellors tended to utilize counselling therapy more than psychologists.

Our research further explored whether a clinicians' therapeutic preference varied according to their country of origin. A comparison of the top five therapies with the top five countries of origin represented found significant differences. The top five therapies that clinicians reported using has been reported in Table 3. Clinicians in Australia used ACT more frequently than clinicians in other countries $\left[X^{2}(d f=4, \mathrm{~N}=91)=18.786, p<0.001\right]$. However, there was consistency across the other four of the five countries in terms of therapy use.

\section{Free Text Responses}

A total of 85 clinicians provided free text comments regarding the challenging and rewarding aspects of working with women with PPP. From the clinicians' perspective, key difficulties included the adjustment from acute to chronic therapeutic approaches, the long-term nature of interventions for women with CPP, and the emotional fatigue experienced when working with women suffering co-morbid physical and mental health conditions. There was concern regarding the prolonged waiting times that their patients' experienced when accessing diagnostic and treatment services. Maintaining communication across a patients' team of treating professionals was also a challenge, particularly for those working out of private settings.

Clinicians reported a number of treatment issues. They reported that clients had a tendency to focus on short term medical approaches that were potentially curative for their condition. They reported that this impaired their ability to engage with therapies that might be more effective for them following appropriate medical intervention, such as long-term management approaches or to view mental health intervention. The clinicians reported that their clients often struggled with challenging pain-related beliefs, avoidance and fear of pain, maladaptive coping skills, and preoccupation with pain and difficulties with acceptance. They also reported that providing emotional support to clients who were distressed, uncomfortable and cognitively rigid was personally exhausting.

In contrast, clinicians also expressed the numerous rewards of working with women with PPP. These included the experience of seeing women gain confidence in managing their pain, regain control over their lives, increased valued activities and improved quality of life. PPP offered clinicians the opportunity to contribute positively to women's lives as part of a collaborative approach, validating their experiences and supporting them towards personal empowerment and confidence. They described finding it rewarding when clients developed insights or progressed towards their pain management goals.

\section{Future Research Priorities}

Multiple future research priorities were identified by the clinicians. First, they wanted more evidence for specific psychological techniques and therapies for females with PPP. Future research into CBT, ACT, mindfulness, psychosexual therapy and hypnosis, trauma prevalence and therapies was of particular interest. Second, clinicians were interested in finding out whether personality traits and distress tolerance were effective predictors of therapy engagement and outcomes. If these factors did predict outcomes, then therapies or techniques targeting these aspects might be effective additions to therapy for women with PPP. Third, clinicians requested more evidence as the most effective psycho-education would be for women with PPP. Specifically, they wanted to know more about the overlap between the biological components of chronic pain and mental health. Finally, clinicians were interested as to whether outcomes were influenced by professional attitudes to PPP. 


\section{Discussion}

This study achieved its key aim of describing the current clinical practices used by mental health clinicians when treating women with PPP. It has reported key findings regarding the therapies and therapies and techniques that clinicians working in this area use, view as evidence based and effective. It also reported clinician experiences and preferences for further research into mental health intervention for women with PPP.

\section{Assessment and the Use of Questionnaires}

Clinicians most commonly assessed for Depression, anxiety, pain catastrophizing and pain related self-efficacy. Clinicians are therefore choosing questionnaires that assess factors known to influence aspects of pain, but also outcomes of mental health interventions. Depression and anxiety have been shown to be positive predictors of symptom severity and pain intensity in women with PPP. ${ }^{38}$ They have also been shown to predict the effectiveness and outcomes of psychological interventions used for chronic pain clients, such as CBT and $\mathrm{ACT}^{39}$ Pain catastrophizing has been shown to be a positive predictor of pain-related disability, self-efficacy in relation to managing pain, difficulties processing pain-related beliefs and emotions. ${ }^{40,41}$ Catastrophizing has been shown to moderate pain intensity and pain medication use in chronic pain groups. ${ }^{40}$ Pain-related beliefs have the potential to influence self-efficacy and treatment adherence in other chronic pain groups. ${ }^{42}$ Self-efficacy with reference to the management of pain has been shown to predict quality of life and pain perceptions reported by clients with chronic lower back pain. ${ }^{41}$ The clinicians appear to be using assessment measures to identify known predictors of mental health outcomes in other chronic pain conditions, so that they can then select appropriate therapies. However, the large range of measures that they reported using, demonstrates a lack of consensus as to what questionnaires were best used for this purpose with women who have PPP.

\section{The Use of Education Concepts in Consultations}

The majority of clinicians in our study included pain education in their sessions and provided additional resources to back up the psycho-education components they provided during sessions. Education about pain and the modulation of pain in the brain improves the effectiveness of physical therapy, therapy engagement, pain perception, coping skills, fear of movement and catastrophizing. ${ }^{43-46}$ However, although pain and disability improved with pain education with moderate effect sizes, Marris et al. ${ }^{45}$ found that the results lacked pooled statistical significance due to differences in included pain-related concepts provided, varied pain severity ratings across study groups, and the chronic pain conditions included. Clinicians are therefore covering education concepts with their female clients with PPP in order to try and enhance therapy engagement and outcomes. However, further research is required in order to establish which specific education components improve outcomes for women with PPP.

\section{Psychological Therapies and Techniques Utilized}

Our study found that Cognitive Behavioral Therapy (CBT) and Acceptance and Commitment Therapy (ACT) were the most common therapies used by clinicians. This provides a clear indication that clinicians were drawing the therapies and techniques that they used from the chronic pain evidence base. This is consistent in their use of techniques such as mindfulness exercises, thought challenging, anxiety and pain belief balancing. Despite most participants initially reporting that they believed that the strength of evidence for mental health intervention for women with PPP was average to strong, almost a third reported that they were unsure which intervention was supported by evidence. Systematic reviews in this area have all concluded that the evidence for mental health interventions and techniques for use with women with PPP conditions is weak. ${ }^{11-13}$ Most clinicians used ACT and mindfulness techniques in this study, techniques that are increasingly used. This is despite a recent meta-analysis finding that effect sizes for use are small to moderate and are not regarded as superior to CBT with reference to chronic pain groups. ${ }^{23}$ It appears that mental health clinicians are choosing to use therapies that are evidence based for improving pain and mental health outcomes, such as depression and anxiety, or other chronic pain conditions. ${ }^{17,22,23}$ However, the clinicians' lack of reported knowledge on the evidence base is problematic, as the evidence for effectiveness of mental health interventions varies according to chronic pain condition.

\section{Co-Morbid Mental Health Conditions}

Participants in our study reported that GAD, MDD, and PTSD were the most common co-morbid conditions treated. Consistent with this finding, clinicians reported the use of CBT and ACT, which have good evidence for use with GAD and MDD. ${ }^{43,44,47,48}$ However, very few clinicians reported the 
use of eye movement desensitization and reprocessing (EMDR), one of two available "gold-standard" therapeutic interventions targeting PTSD symptoms. This is supported by the qualitative findings that clinicians wanted to know more about the prevalence of trauma and PTSD with reference to women with PPP, and how to treat these aspects in this group. Evidence suggests that people with chronic pain syndromes are four times more likely to be at risk of developing PTSD and report more functional impact than those without a chronic pain condition. ${ }^{49}$ Women are estimated to have twice the risk of developing PTSD than men. ${ }^{50}$ Although poorer health outcomes have been reported for women with PPP and PTSD diagnoses than women with PPP without PTSD, it is difficult to establish whether women with PPP have increased prevalence of PTSD or traumatic experiences in comparison to the general population. ${ }^{51}$ There is however, evidence to suggest that they have high rates of anxiety and depression in comparison to the general population. ${ }^{52}$

\section{Relaxation Techniques Utilized}

Progressive muscle relaxation, diaphragmatic breathing and relaxation breathing were the most common techniques used by clinicians. In the absence of studies on relaxation for women with PPP, clinicians are drawing from evidence for relaxation in other chronic pain groups. Recent reviews on relaxation for chronic pain show that progressive muscle relaxation can reduce pain intensity in patients with arthritis and lower back pain. ${ }^{53}$ However, these positive outcomes were not sustained at follow up after six months. ${ }^{53}$ A recent systematic review by Jeffrey et $\mathrm{al}^{54}$ concluded that there was no evidence for the use of relaxation as a stand-alone therapy to reduce outcomes such as pain intensity and poor quality of life in the longer term. However, results from a study by Zhao et al ${ }^{55}$ suggested that progressive muscle relaxation lead to reduced anxiety, reduced depression, and improved quality of life in a sample of 50 women with endometriosis. Such exercises have also been shown to lead to improvements in anxiety and depression for groups with lower back pain and IBS. ${ }^{56}$ Pelvic muscle relaxation and "reprogramming" the pelvic floor using physiotherapy techniques is effective for chronic pelvic pain. ${ }^{57}$ However, it is yet to be established whether muscle-based relaxation or other forms of relaxation are effective for women with PPP when administered by mental health clinicians.

\section{The Role of Hypnosis}

Hypnosis has been demonstrated to be effective in other chronic conditions such asIBS and those with lower back pain. ${ }^{58,59} \mathrm{~A}$ recent study by Vanhaudenhuyse et $\mathrm{al}^{43}$ found that the use of hypnosis in conjunction with pain education and therapy led to reductions in problematic pain-related beliefs and improved coping for clients with chronic pain conditions. Improvements in pain-related outcomes appears to be dependent on suggestibility and the use of analgesic suggestions. ${ }^{58,59}$ Our study found that few of the clinicians surveyed used hypnosis when managing PPP. At present, the sparse literature available is insufficient evidence for the use of hypnosis with females who have PPP either as an adjunct to a mental health intervention or as a stand-alone protocol. This may be why clinicians in this study did not report the use of hypnosis with their clients, despite the evidence base for its' use in other chronic conditions.

\section{Differences in Therapy Use Across Profession and Country}

The psychologists in our study reported a preference for the use of CBT and ACT. This reflects the current evidence base for the use of psychology with other chronic pain conditions, or the training that clinicians have received. As CBT has a strong evidence base, it is taught within undergraduate and postgraduate psychology training programs. ${ }^{60} \mathrm{ACT}$ is often taken up in post-Masters training and has emerging evidence for effectiveness in other chronic pain groups. However, some clinicians appear to have a strong preference for ACT despite the evidence base for CBT. Therefore further research is required to establish why this preference exists and which is best for PPP. Counselling interventions have specific features and are more frequently studied within counselling Masters programs. They include a number of different techniques and interventions, and therefore it is unclear what exactly they are using in sessions and whether it is evidence-based.

The causal factors behind the unexpected geographical variation in treatment preference require further investigation particularly with regard to patient outcome. Australian clinicians were more likely to use ACT therapies than their counterparts internationally. If clinicians were practicing according to the evidence within international literature, uniformity in approaches used across geographic locations should be anticipated, but this was not supported by our research findings. This geographical difference may reflect the relevant availability of psychology training available in Australia over the last 5-10 years. It may also reflect clinician preference or an alignment between the therapeutic components of ACT and Australian cultural preference. Research by Varra et $\mathrm{al}^{61}$ reported that when clinicians found ACT useful for their own learning and 
psychological flexibility, they were more likely to adopt it for use with their clients.

\section{Experiences of the Clinicians Working with Women Who Have PPP}

Our research describes the challenges commonly reported by clinicians working with women with PPP. These included client adjustment to the chronic pain approach, service access limitations, and working with co-morbid health and mental health conditions. Clinicians reported sometimes feeling exhausted by the prospect of working with these client challenges. This is an experience also reported by clinicians managing other chronic pain conditions. A study of clinicians caring for chronic pain affected veterans by Dobscha et $\mathrm{al}^{62}$ found that although while clinicians were confident and interested in assisting their clients, they reported low levels of workplace satisfaction due to a perceived inability to provide optimal treatment and inadequate support. Militello et al ${ }^{63}$ interviewed primary care clinicians working with chronic pain clients and described a reported difficulty in determining the treatment components to include in their patients' care. They recommended the development of evidence-based and collaborative frameworks for decision-making with reference to pain clients, which were both convenient for clinicians and considerate of social and environmental factors. Our research suggests that may require further support to assist them when working with women who have PPP.

\section{Future Research Directions Identified by Clinicians}

Confusion remains regarding the efficacy of CBT, ACT, mindfulness, PTSD and hypnosis for treating women with PPP, and the need for further research was confirmed. Clinicians were significantly interested in understanding the overlap between the mental and physical aspects of PPP, including the biology, neuroscience, co-occurring physical conditions, personality traits and distress tolerance of their patients. They wanted to know more about whether client outcomes were influenced by professional attitudes to PPP, and which techniques should be included within clinician training programs. Further investigations as to why clinicians choose the therapies would be beneficial. While few clinicians reported using hypnotic intervention in this study, they identified hypnosis as an area of interest for further research. This likely reflects the increasing evidence for hypnosis in improving outcomes such as pain related beliefs and coping skills in other chronic pain groups. ${ }^{59}$

\section{Limitations}

Our research has limitations in its application. Although we identified the types of therapies that clinicians used, we did not investigate the content or particular aspects of these techniques that they preferred. We also did not provide definitions of each therapy listed in our survey choice options, which would have contributed to standardization. Our sample size was small. For instance, $28 \%$ of the sample was in "other" category in terms of profession. However, this is testament to the breadth of professions working in mental health and as working with women who have PPP is a niche field of practice. Though we endeavored to capture a representative sample, not all mental health clinicians are listed on professional organization sites or publically listed. Participants were mostly female, used English as a key language, were information technology literate, and worked in a private clinical setting. For these reasons, our results may not be generalizable to other clinical settings or within diverse cultures.

\section{Conclusion}

An evidence base for psychological interventions for women with PPP is desperately needed to inform clinical practice in mental health and to improve therapy outcomes. Mental health clinicians currently use psychological interventions and techniques with women who have PPP which are only evidenced for chronic pain in general. CBT and ACT were the most commonly used therapies, which may prove effective given the overlap seen in COPCs. However, current clinical practice across disciplines and countries does not appear uniform, despite being drawn from the same evidence base. There is also limited understanding as to how mental health interventions need to be tailored to address the aspects of PPP that may be unique in comparison to other COPCs. There is a distinct need for further evidence to support clinical practice in this area. The key therapies and techniques identified by clinicians as requiring further research were, $\mathrm{CBT}$, ACT, mindfulness, trauma therapy, hypnosis, personality factors and distress tolerance. Despite finding working with women who had PPP rewarding, clinicians reported challenges to therapy including fatigue and the long-term nature of support required. This highlights for a specific evidence-based intervention consensus for the effective treatment of PPP that considers clinical perspectives and needs. 


\section{Acknowledgments}

The authors would like to acknowledge the support of the Pelvic Pain Foundation of Australia, Australian Psychological Society, and all the clinicians that participants that donated their time to this study. We would also like to thank Professor Lorimer Mosely for his contribution to validity testing for this study.

\section{Funding}

This project received no funding.

\section{Disclosure}

Dr Susan Evans reports is chair from Pelvic Pain Foundation of Australia, a shareholder and board member of Alyra Biotech developing novel treatments for pelvic pain. from Alyra Biotech Pty Ltd, is author of the book "Endometriosis and Pelvic Pain" which is sold to members of the public with pelvic pain from Dr Susan F Evans Pty Ltd, outside the submitted work; and provided paid educational presentations for Bayer and Pfizer. The authors report no other conflicts of interest in this work.

\section{References}

1. Ahangari A. Prevalence of chronic pelvic pain among women: an updated review. Pain Physician. 2014;17(2):e141-7. doi:10.36076/ ppj.2014/17/E141

2. Nicholas M, Vlaeyen JWS, Rief W, et al. IASP taskforce for the classification of chronic pain. The IASP classification of chronic pain for ICD-11: chronic primary pain. Pain. 2019;160(1):28-37. doi:10.1097/j.pain.0000000000001390

3. Till SR, As-Sainie S, Schrepf A. Psychology of chronic pelvic pain: prevalence, neurobiological vulnerabilities, and treatment. Clin Obstet Gynaecol. 2019;62(1):22-36.

4. Armour M, Lawson K, Wood A, Smith CA, Abbott J. The cost of illness and economic burden of endometriosis and chronic pelvic pain in Australia: a national online survey. PLoS One. 2019;14(10): e0223316. doi:10.1371/journal.pone.0223316

5. Da Luz RA, de Deus JM, Conde DM. Quality of life and associated factors in Brazilian women with chronic pelvic pain. $J$ Pain Res. 2018;11:1367-1374. doi:10.2147/JPR.S168402

6. Siqueira-Campos VME, Da Luz RA, de Deus JM, Martinez EZ, Conde DM. Anxiety and depression in women with and without chronic pelvic pain: prevalence and associated factors. J Pain Res. 2019;12:1223-1233. doi:10.2147/JPR.S195317

7. Brunahl C, Dybowski C, Albrecht R, et al. Mental disorders in patients with chronic pelvic pain syndrome. J Psychosom Res. 2017;98:19-26. doi:10.1016/j.jpsychores.2017.04.011

8. Pain treatment services [homepage on the internet]. International Association for the study of pain; 2020. Available from: www.iasppain.org/Education/Content.aspx?ItemNumber=1381. Accessed December 12, 2020.

9. Australian Pain Society position statement [homepage on the internet]. Australian Pain Society; 2016. Available from: https://www.apsoc.org.au/ PDF/Position_Paper/20160321_APS_Psychology_Position_Statement_ MAR16.pdf. Accessed December 5, 2020.
10. Beales D, Slater H, Palsson T, Sullivan PO. Understanding and managing pelvic girdle pain from a person-centred biopsychosocial perspective. Musculoskelet Sci Prac. 2020;48:102152. doi:10.1016/j. msksp.2020.102152

11. Stones W, Cheong YC, Howard FM, Singh S. Interventions for treating chronic pelvic pain in women. Cochrane Database of Syst Rev. 2005;2.

12. Van Niekerk L, Weaver-Pirie B, Matthewson M. Psychological interventions for endometriosis related symptoms: a systematic review with narrative data synthesis. Arch Womens Ment Health. 2019;22 (6):723-735. doi:10.1007/s00737-019-00972-6

13. Andrews J, Yunker A, Reynolds WS, Likis FE, Sathe NA, Jerome RN. Noncyclic Chronic Pelvic Pain Therapies for Women: Comparative Effectiveness. Rockville (MD): Agency for Healthcare Research and Quality, Vol. 11; 2012: EHC088-EFAvailable from https://www.ncbi. nlm.nih.gov/books/NBK84586/. Accessed June 17, 2021.

14. Till SR, As-Sanie S, Schrepf A. Psychology of chronic pelvic pain: prevalence, neurobiological vulnerabilities, and treatment. Clin Obstet Gynecol. 2019;62(1):22-36. doi:10.1097/GRF.0000000000000412

15. Malfiet A, Kregal J, Coppieters I, et al. Effect of pain neuroscience education combined with cognition-targeted motor control training on chronic spinal pain: a Randomized Clinical Trial. JAMA Neurol. 2018;75(7):808-817. doi:10.1001/jamaneurol.2018.0492

16. Zidarov D, Visca R, Ahmed S. Type of clinical outcomes used by healthcare professionals to evaluate health-related quality of life domains to inform clinical decision making for chronic pain management. Qual Life Res. 2019;28(10):2761-2771. doi:10.1007/ s11136-019-02215-8

17. Ehde DM, Dillworth TM, Turner JA. Cognitive-behavioral therapy for individuals with chronic pain: efficacy, innovations, and directions for research. Am Psychologist. 2014;69(2):153-166. doi:10.1037/a0035747

18. Richmond H, Hall AM, Copsey B, et al. The Effectiveness of Cognitive Behavioural Treatment for Non-Specific Low Back Pain: a Systematic Review and Meta-Analysis. PLoS One. 2015;10(8): e0134192. doi:10.1371/journal.pone.0134192

19. Alappattu MJ, Bishop MD. Psychological factors in chronic pelvic pain in women: relevance and application of the fear-avoidance model of pain. Phys Ther. 2011;91(10):1542-1550. doi:10.2522/ ptj.20100368

20. Dillworth T, Jensen MP. The role of suggestions in hypnosis for chronic pain: a Review of the Literature. Open Pain J. 2010;3 (1):39-51. doi:10.2174/1876386301003010039

21. Elkins G, Jensen MP, Patterson DR. Hypnotherapy for the management of chronic pain. Int J Clin Exp Hypn. 2007;55(3):275-287. doi:10.1080/00207140701338621

22. Hughes LS, Clark J, Colclough JA, Dale E, Mcmillan D. Acceptance and commitment therapy ACT for chronic pain: a systematic review and meta-analyses. Clin J Pain. 2017;33(6):552-568. doi:10.1097/ AJP. 0000000000000425

23. Veehof MM, Trompetter HR, Bohlmeijer ET, Schreurs KM. Acceptance and mindfulness based interventions for the treatment of chronic pain: a meta-analytic review. Cogn Behav Ther. 2016;45 (1):5-31. doi:10.1080/16506073.2015.1098724

24. Veasley C, Clare D, Clauw DJ, et al. Impact of chronic overlapping pain conditions on public health and the urgent need for safe and effective treatment: 2015 analysis and policy recommendations. CPRA. 2015.

25. Maixner W, Fillingim RB, Williams DA, Smith SB, Slade GD. Overlapping chronic pain conditions: implications for diagnosis and classification. J Pain. 2016;17(9 Suppl):T93-T107. doi:10.1016/j. jpain.2016.06.002

26. Arendt-Nielson L, Morlion B, Perrot S, et al. Assessment and manifestation of central sensitization across different chronic pain conditions. Eur J Pain. 2018;22:216-241.

27. Yosef A, Ghaffar A, Al Hussani T, Abdellah MS, Cue G, Bedaiwy MA. Chronic pelvic pain: pathogenesis and validated assessment. Middle East Fertility Soc J. 2016;21(4):205-221. doi:10.1016/j.mefs.2016.08.001 
28. Kumar R, Scott K. Chronic pelvic pain and the chronic overlapping pain conditions in women. Curr Phys Med Rehab Rep. 2020;8 (3):207-216. doi:10.1007/s40141-020-00267-3

29. McGowan L, Escott D, Luker K, Creed F, Chew-Graham C. Is chronic pelvic pain a comfortable diagnosis for primary care practitioners: a qualitative study. BMC Fam Pract. 2010;11(1):7. doi:10.1186/1471-2296-11-7

30. Matthias MS, Parpart AL, Nyland KA, et al. The patient-provider relationship in chronic pain care: providers' perspectives. Pain Med. 2010;11(11):1688-1697. doi:10.1111/j.15264637.2010.00980.x

31. SurveyMonkey online survey software [homepage on the internet]. Survey Monkey; 2020. Available from: https://www.surveymonkey. com/. Accessed July 3, 2020.

32. MedCalc Software. MedCalc Statistical Software Version 16. 4. 3. Ostend: Belgium; 2016.

33. IBM Corp. IBM SPSS Statistics for Windows Version 26.0. New York; 2019

34. Lovibond SH, Lovibond PF. Manual for the Depression Anxiety Stress Scales. 2nd ed. Sydney: Psychology Foundation; 1995.

35. Sullivan MJL, Bishop SR, Pivik J. The pain catastrophizing scale: development and validation. Psychol Assess. 1995;7(4):524-532. doi:10.1037/1040-3590.7.4.524

36. Nicholas MK. The pain self-efficacy questionnaire: taking pain into account. Europ $J$ Pain. 2007;11(2):153-163. doi:10.1016/j. ejpain.2005.12.008

37. Evidenced based multimedia resources for the treatment of pain [homepage on the internet]. NOI Group; 2020). Available from: https://www.noigroup.com/. Accessed November 11, 2020.

38. Dybowski C, Lowe B, Brunahl C. Predictors of pain, urinary symptoms and quality of life in patients with chronic pelvic pain syndrome (CPPS): a prospective 12-month follow-up study. J Psychsom Res. 2018;112:99-106. doi:10.1016/j.jpsychores.2018.06.013

39. Gilpin HR, Keyes A, Sttahl DR, Grieg R, McCracken LM. Predictors of treatment outcome in contextual cognitive and behavioural therapies for chronic pain: a systematic review. J Pain. 2017;18 (10):1153-1164. doi:10.1016/j.jpain.2017.04.003

40. Leung L. Pain catastrophizing: an updated review. Indian J Psychol Med. 2012;34(3):204-217. doi:10.4103/0253-7176.106012

41. Yazdi-Ravandi S, Taslimi Z, Jamshidian N, Saber H, Shams J, Abbas H. Prediction of quality of life by self-efficacy, pain intensity and pain duration in patient with pain disorders. Basic Clin Neurosci. 2013;4:11-18.

42. Flaskerud JH. Pain and culture: the catastrophizing construct and measurement issues in mental health nursing. Issues Ment Health Nurs. 2015;36(2):152-155. doi:10.3109/01612840.2014.941078

43. Vanhaudenhuyse A, Gillet A, Malaise N, et al. Psychological interventions influence patients' attitudes and beliefs about their chronic pain. J Tradit Complement Med. 2018;8(2):296-302. doi:10.1016/j. jtcme.2016.09.001

44. Darlow B, Dowell A, Baxter GD, Mathieson F, Perry M, Dean S. The enduring impact of what clinicians say to people with low back pain. Ann Fam Med. 2013;11(6):527-534. doi:10.1370/afm.1518

45. Marris D, Theophanous K, Cabezon P, Dunlap Z, Donaldson M. The impact of combining pain education strategies with physical therapy interventions for patients with chronic pain: a systematic review and meta-analysis of randomized controlled trials. Physiother Theory Pract. 2019;28:1-12.

46. Watson JA, Ryan CG, Cooper L, et al. Pain neuroscience education for adults with musculoskeletal pain: a mixed-methods systematic review and meta-analysis. J Pain. 2019;20(10):p1140.e1-1140.e22. doi:10.1016/j.jpain.2019.02.011

47. Butler AC, Chapman JE, Forman EM, Beck AT. The empirical status of cognitive-behavioural therapy: a review of meta-analyses. Clin Psych Rev. 2006;26(1):17-31. doi:10.1016/j.cpr.2005.07.003
48. Newby JM, McKinnon A, Kuyken W, Gilbody S, Dalgeleish T. Systematic review and meta-analysis of trans-diagnostic psychological treatments for anxiety and depressive disorders in adulthood. Clin Psych Rev. 2015;40:91-110. doi:10.1016/j.cpr.2015.06.002

49. Twohig MP, Levin ME. Acceptance and commitment therapy as a treatment for anxiety and depression: a review. Psychiatr Clin North Am. 2017;40(4):751-770. doi:10.1016/j.psc.2017.08.009

50. Zhenggang B, Luo S, Zhang L, Wu S, Chi I. Acceptance and Commitment Therapy (ACT) to reduce depression: a systematic review and meta-analysis. $J$ Affect Disord. 2020;260:728-737. doi:10.1016/j.jad.2019.09.040

51. McWilliams LA, Cox BJ, Enns MW. Mood and anxiety disorders associated with chronic pain: an examination in a nationally representative sample. Pain. 2003;106(1):127-133. doi:10.1016/S03043959(03)00301-4

52. Stress and trauma [homepage on the internet]. Australian Institute Health and Welfare;2020. Available from: https://www.aihw.gov.au/reports/aus tralias-health/stress-and-trauma. Accessed November 1, 2020.

53. Kwekkeboom KL, Gretarsdottir E. Systematic Review of relaxation interventions for pain. J Nurs Scholarsh. 2006;38(3):269-277. doi:10.1111/j.1547-5069.2006.00113.x

54. Jeffrey S, McClelland T, Carus C, Graham C. Relaxation and chronic pain: a critical review. Int $J$ Ther Rehabil. 2016;23(6):1759-1779. doi:10.12968/ijtr.2016.23.6.289

55. Zhao L, Wu H, Zhou X, Wang Q, Zhu W, Chen J. Effects of progressive muscular relaxation training on anxiety, depression and quality of life of endometriosis patients under gonadotrophin-releasing hormone agonist therapy. Eur J Obstet Gynecol Reprod Biol. 2012;162(2):211-215. doi:10.1016/j.ejogrb.2012.02.029

56. Bordoni B, Morabito B. Symptomatology correlations between the diaphragm and irritable bowel syndrome. Cureus. 2018;10(7):e3036.

57. Anderson RU, Wise D, Nathanson BH. Chronic prostatitis and/or chronic pelvic pain as a psycho-neuromuscular disorder: a meta-analysis. Urology. 2018;120:23-29. doi:10.1016/j. urology.2018.07.022

58. Lee HH, Choi YY, Choi MG. The efficacy of hypnotherapy in the treatment of irritable bowel syndrome: a Systematic Review and Meta-analysis. J Neurogastroenterol Motil. 2014;20(2):152-162. doi:10.5056/jnm.2014.20.2.152

59. Thompson T, Terhune DB, Oram C, et al. The effectiveness of hypnosis for pain relief: a systematic review and meta-analysis of 85 controlled experimental trials. Neurosci Biobehav Rev. 2019;99:298-310. doi:10.1016/j.neubiorev.2019.02.013

60. Accreditation programs for psychology programs. Australian Psychological Accreditation Council (APAC);2019. Available from: https://www.psychologycouncil.org.au/sites/default/files/public/ APAC_Accreditation_Standards_2018_Jan_Version_for_Online_ Publishing_Single.pd. Accessed November 11, 2020.

61. Varra AA, Hayes SC, Roget N, Fisher G. A randomized control trial examining the effect of acceptance and commitment training on clinician willingness to use evidence-based pharmacotherapy. J Consult Clin Psychol. 2008;76(3):449-458. doi:10.1037/0022006X.76.3.449

62. Dobscha SK, Corson K, Flores JA, Tansill EC, Gerrity MS. Veterans affairs primary care clinicians' attitudes toward chronic pain and correlates of opioid prescribing rates. Pain Med. 2008;9 (5):564-571. doi:10.1111/j.1526-4637.2007.00330.x

63. Militello LG, Anders S, Downs SM, et al. Understanding how primary care clinicians make sense of chronic pain. Cogn Tech Work. 2018;20(4):575-584. doi:10.1007/s10111-018-0491-1 


\section{Publish your work in this journal}

The Journal of Multidisciplinary Healthcare is an international, peerreviewed open-access journal that aims to represent and publish research in healthcare areas delivered by practitioners of different disciplines. This includes studies and reviews conducted by multidisciplinary teams as well as research which evaluates the results or conduct of such teams or healthcare processes in general. The journal covers a very wide range of areas and welcomes submissions from practitioners at all levels, from all over the world. The manuscript management system is completely online and includes a very quick and fair peer-review system. Visit http://www.dovepress.com/testimonials. php to read real quotes from published authors. 